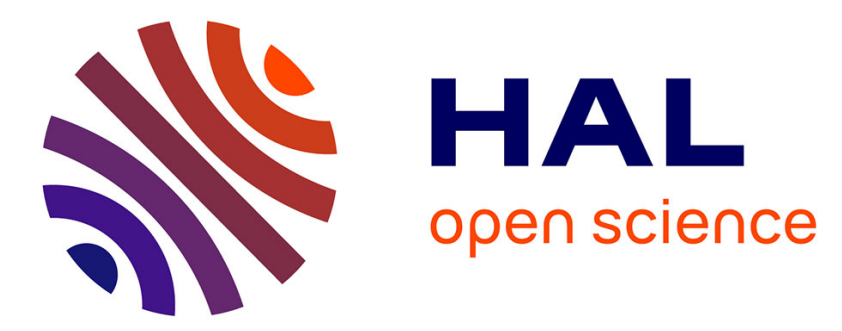

\title{
Local energy approach to steel fatigue
}

Andre Chrysochoos, B. Berthel, Félix Latourte, Stéphane Pagano, Bertrand

Wattrisse, B. Weber

\section{To cite this version:}

Andre Chrysochoos, B. Berthel, Félix Latourte, Stéphane Pagano, Bertrand Wattrisse, et al.. Local energy approach to steel fatigue. Strain, 2008, 44 (4), pp.327-334. 10.1111/j.1475-1305.2007.00381.x . hal-00447351

\section{HAL Id: hal-00447351 \\ https://hal.science/hal-00447351}

Submitted on 24 Sep 2021

HAL is a multi-disciplinary open access archive for the deposit and dissemination of scientific research documents, whether they are published or not. The documents may come from teaching and research institutions in France or abroad, or from public or private research centers.
L'archive ouverte pluridisciplinaire HAL, est destinée au dépôt et à la diffusion de documents scientifiques de niveau recherche, publiés ou non, émanant des établissements d'enseignement et de recherche français ou étrangers, des laboratoires publics ou privés. 


\title{
Local Energy Approach to Steel Fatigue
}

\author{
A. Chrysochoos*, B. Berthel*t, F. Latourte*, S. Pagano*, B. Wattrisse* and B. Weber ${ }^{\dagger}$ \\ *Laboratory of Mechanics and Civil Engineering, CC 048, PI. E. Bataillon, 34095 Montpellier, France \\ ${ }^{\dagger}$ Arcelor Research SA, Voie Romaine BP 30320, 57283 Maizières-lès-Metz, France
}

\begin{abstract}
This paper presents an experimental protocol developed to locally estimate different terms of the energy balance associated with the fatigue of DP600 steel. The method involves two quantitative imaging techniques. First, digital image correlation provides displacement fields and, after derivation, strain and strain-rate fields. A variational method, associated with an energy functional, is used to simultaneously identify elastic parameter and stress fields. The deformation energy rate distribution can then be determined on the basis of the stress and strain data. Secondly, infrared thermography provides thermal images which are used to separately estimate the thermoelastic source amplitude and mean dissipation per cycle distributions. The image processing uses a local form of the heat diffusion equation and a special set of approximation functions that take the frequency spectra of the sought sources into account.
\end{abstract}

KEY WORDS: digital image correlation, dissipation, fatigue, infrared thermography, steel

\section{Introduction}

Characterisation of fatigue in materials and mechanical components requires time-consuming and expensive statistical processing of the results of numerous mechanical tests. Over the last two decades, alternative experimental approaches have been developed with reliable fatigue characteristics, including thermal methods based on the analysis of self-heating during stepwise loading fatigue tests [1-6]. Indeed, during high cycle fatigue of steel specimens, the stress states traditionally remain within the mesoscopic elastic domain of the material. However, slight energy dissipation caused by irreversible microstructural changes is observed during the many cycles performed at high loading frequencies. This dissipation is then combined with the standard thermoelastic coupling caused by thermal expansion of the crystalline network. In the references mentioned above, several authors claimed that the remarkable change in heating regime that occurs within a certain stress range is empirically related to the fatigue limit of the material. Although realistic estimates of this limit have sometimes been obtained, the thermal approach often leads to questionable results. Indeed, the physical implications of the threshold stress evaluated by these thermal methods are not well understood. In Ref. [7], it was shown that the onset of persistent slip bands was associated with a loss of linearity of the temperature versus stress range correspondence. It was also shown that the persistent slip band number increased with stress and seemed to be a relevant fatigue indicator.

The direct use of temperature as a fatigue indicator is not always reliable because the temperature variation is not intrinsic to the material behaviour [8]. It actually depends on the diffusion properties along with the thermal boundary conditions and heat source distribution. Here we therefore studied fatigue phenomena using a local energy approach with the aim of experimentally determining the deformation energy rate distribution and the distribution of various heat sources accompanying the fatigue test.

Speckle image correlation techniques, involving a digital charge-coupled device (CCD) camera [9], were used to assess surface displacement fields. These kinematic fields enabled us to identify distributions of elastic parameters and stress patterns by minimising a given energy functional. A local form of the heat equation was also used to derive heat sources from thermal images provided by an infrared focal plane array (IRFPA) camera. A specific thermal image processing method was therefore developed to separately estimate dissipated energy and thermoelastic coupling sources.

In what follows, we first review the form of the different terms of the energy balance associated with high cycle fatigue of steels. We then briefly review kinematic and thermal image-processing principles 
and the benefits of their combined use. Finally, preliminary results obtained on dual-phase steel are presented to highlight the potential of this local energy approach.

\section{Local Form of the Energy Balance}

From a thermomechanical standpoint, fatigue is considered as a dissipative quasi-static process. Within the generalised standard material framework [10], the equilibrium state of each volume material element is then described using a set of $N$ state variables. The chosen variables are: the absolute temperature $T$, the linearised strain tensor $\varepsilon$ (hypothesis of small perturbations) and $N-2$ scalar components $\alpha_{1}, \alpha_{2}, \ldots, \alpha_{N-2}$ of vector $\alpha$ which pools the so-called internal variables. These latter variables describe the macroscopic effects of complex coupled microstructural phenomena.

The rate of deformation energy $w_{\text {def }}^{\bullet}$ is classically defined by:

$w_{\mathrm{def}}^{\bullet}=\sigma: \dot{\varepsilon}$

where $\sigma$ is the Cauchy stress tensor. The symbol ( ) means that the time variation of ( ) is path-dependent.

The local heat diffusion equation is written as:

$\rho C \dot{T}-\operatorname{div}(\operatorname{Kgrad} T)=d_{1}+s_{\text {the }}+s_{\text {ic }}+r_{\text {ext }}$

where $\rho$ is the mass density, $C$ the specific heat and $K$ the conduction tensor. The heat sources are, in turn, the intrinsic dissipation $d_{1}$, the thermomechanical coupling sources that pool the thermoelastic source $s_{\text {the }}$ and the other internal coupling sources $s_{\mathrm{ic}}$, and the external volume heat supply $r_{\text {ext }}$. With the specific free energy $\psi(T, \varepsilon, \alpha)$, the volume heat sources $d_{1}$, $s_{\text {the }}$ and $s_{\text {ic }}$ can be rewritten as:

$d_{1}=\sigma: \dot{\varepsilon}-\rho \partial \psi / \partial \varepsilon \cdot \dot{\varepsilon}-\rho \partial \psi / \partial \alpha \cdot \dot{\alpha}$

$s_{\text {the }}+s_{\text {ic }}=\rho T \partial^{2} \psi / \partial T \partial \varepsilon: \dot{\varepsilon}+\rho T \partial^{2} \psi / \partial T \partial \alpha \cdot \dot{\alpha}$

In the framework of our fatigue tests, temperature variations remained minimal and could not modify the internal state of the material, so we neglected the corresponding heat sources $s_{\text {ic }}$ and the thermoelastic sources were computed via the classical linear thermoelastic model applied to uniaxial loadings. The thermoelastic source could then be written as $s_{\text {the }} \approx-\alpha_{\mathrm{d}} T_{0} \dot{\sigma}$, where $\alpha_{\mathrm{d}}$ is the linear thermal expansion coefficient.

\section{DIC and IRT Image Processing}

\section{Digital image correlation}

Digital image correlation (DIC) gives the space-time patterns of various kinematic variables on the sample surface [9] (displacement, velocity, strain, strain-rate, acceleration, etc.). The camera must be carefully set so that the CCD detector remains parallel to the sample surface throughout the test. Indeed, each outof-plane movement (translation or rotation) modifies the image magnification and generates parasitic strains which disturb the kinematic measurements.

In our study, image processing was systematically performed after the test in two steps. First, the displacement field was estimated. Secondly, the strains (or the strain rates) were derived from the displacements by space (and time) differentiation. Each computational step involved a specific numerical processing procedure based on local least squares fitting, as thoroughly presented in Ref. [9]. The image processing performances were tested both on analytic and experimental cases corresponding to rigid body motion (translation or rotation), or to homogeneous or heterogeneous strain. In the case of fatigue tests described hereafter, the accuracy in terms of displacement measurement was about $5 \times 10^{-2}$ pixels and the strain calculation was $1 \times 10^{-4}$. The reader, interested in the metrological issues of the work, can refer to Ref. [9].

To estimate $w_{\text {def }}^{\bullet}$, we used the in-plane displacement field $u=(U, V)$ measured by DIC under the plane stress hypothesis. The overall forces applied at the specimen boundary were recorded. For each loading step, the variational approach provided a couple $(E, \sigma)$ which can provide a solution to the identification problem if it satisfies the local equilibrium equations, linear elasticity equations and global equilibrium.

We associated the functional $F$ defined by Ref. [11] to this problem:

$F(\sigma, B)=\frac{1}{2} \int_{\Omega}(\sigma-B: \varepsilon(u)): B^{-1}:(\sigma-B: \varepsilon(u)) \mathrm{d} \Omega$

where the stress field $\sigma$ is statically admissible, and where the compliance tensor $B$ is supposed to be symmetrical, non-negative and definite. The functional $F(\sigma, B)$ is convex and positive, and null if and only if the couple $(\sigma, B)$ satisfies the constitutive equation. Identification was then performed by numerically minimising the functional $F(\sigma, B)$. A minimisation over the first and second variable gives the stress field solution and the field of piecewise constant elastic parameters, respectively [12]. To 
check the consistency of the results given by the variational approach, plane stress components were also computed by using local equilibrium equations and strain data, as already performed in Refs [13, 14]. Energy estimates shown at the end of the paper, state that both approaches give similar results.

\section{Infrared thermography}

The left-hand side of Equation (2) is a differential operator applied to $T$, while its right-hand side groups all possible heat sources accompanying the deformation process. The regularising effects of heat diffusion limit the thermal gradients throughout the (small) thickness of the specimen (Figure 1). A depthwise averaged heat source distribution can thus usually be estimated by using an integrated form over the sample thickness of the heat equation and by assuming that thermal data on the surface remain close to the average temperature $[15,16]$.

In our experiments, we assumed that the material parameters $\rho, C, K$ were constant. We considered isotropic heat diffusion and neglected the convective terms in the material time derivative of the temperature. We also assumed that the internal coupling sources remain negligible near thermal equilibrium (i.e. no stress-induced phase change). Besides, we verified that the external heat supply (here limited to radiation exchanges) remained time-independent, so that:

$-k \Delta T_{0}=r_{\mathrm{ext}}$

where $k$ is the isotropic conduction coefficient and $T_{0}$ the equilibrium temperature field of the sample. For tests performed on thin flat specimens, it was shown in Ref. [16] that the temperature measured on the surface of the sample remains very close to the depthwise average temperature as long as the time constant characterising the heat diffusion remains small

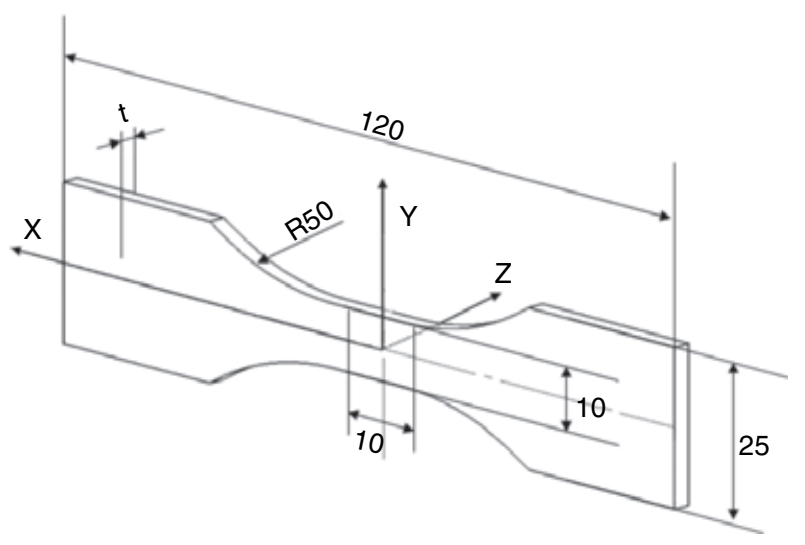

Figure I: Shape of the specimen compared with the loading time period. Taking all of these hypotheses into account, Equation (2) could be markedly simplified into a two-dimensional partial derivative equation:

$\rho C\left(\frac{\partial \bar{\theta}}{\partial t}+\frac{\bar{\theta}}{\tau_{\text {th }}}\right)-k \Delta \bar{\theta}=\bar{d}_{1}+\bar{s}_{\text {the }}$

where $\bar{\theta}=\bar{T}-T_{0}$ is the difference between $T$ and $T_{0}$, averaged according to thickness. The symbol $\tau_{\text {th }}$ stands for a time constant characterising heat losses perpendicular to the plane of the specimen, while heat conduction in the plane is taken into account by the two-dimensional Laplacian operator.

Construction of the heat source distribution using Equation (6) requires evaluation of partial derivative operators applied to noisy digital signals. To compute reliable estimates of heat sources, it is then necessary to reduce the noise amplitude without modifying the spatial and temporal thermal gradients. Among several possible methods, a special local least-squares fitting of the thermal signal was considered hereafter (cf. Equation 9). Approximation functions account for the underlying heat source spectral properties. Moreover, the linearity of Equation (6) and that of the respective boundary conditions enabled us to separately analyse the influence of thermoelastic and dissipative heat sources.

Indeed, within the linear thermoelasticity framework, it is easy to verify that:

- the thermoelastic source has the same frequency spectrum as the stress signal;

- the variation of the thermoelastic energy $w_{\text {the }}$ vanishes at the end of each loading cycle of period $f_{\mathrm{L}}^{-1}$, so we get:

$\tilde{w}_{\text {the }}=\int_{f_{\mathrm{L}}^{-1}} s_{\text {the }} \mathrm{d} t=0$.

Regarding the dissipative effects, we considered dissipation averaged over a whole number $n$ of complete cycles (e.g. $n$ - number of cycles per second or per block):

$\tilde{d}_{1}=\int_{n f_{\mathrm{L}}^{-1}} n^{-1} f_{\mathrm{L}} \bar{d}_{1} \mathrm{~d} t$

The new variable $\tilde{d}_{1}$ may characterise the slow degradation of the material microstructure because of fatigue phenomena including diffuse microplasticity, development of localised persistent slip bands, and progressive damage growth leading to microcracks. The average dissipation per cycle $\tilde{d}_{1}$ is thus a positive heat source whose spectrum is limited to very low 
frequencies. With $\tilde{\Delta} s_{\text {the }}$ denoting the thermoelastic source range averaged over $n$ cycles, the aim of the image processing is then to separately assess $\tilde{\Delta} s_{\text {the }}$ and $\tilde{d}_{1}$.

The local fitting function $\theta^{\mathrm{fit}}$ of the temperature charts is chosen as:

$$
\begin{aligned}
\theta^{\mathrm{fit}}(x, y, t)= & p_{1}(x, y) t+p_{2}(x, y)+p_{3}(x, y) \cos \left(2 \pi f_{\mathrm{L}} t\right) \\
& +p_{4}(x, y) \sin \left(2 \pi f_{\mathrm{L}} t\right)
\end{aligned}
$$

where the trigonometric time functions describe the periodic part of the thermoelastic effects while the linear time function takes transient effects caused by heat loss, dissipative heating and possible drift in the equilibrium temperature into account. Functions $p_{i}(x, y), i=1, \ldots, 4$, are second-order polynomials in $x$ and $y$ [17]. These polynomials enabled us to take possible spatial heterogeneity in the source patterns into account. The local approximation zones are rectangular made of $(2 \times 6+1)$ by $(2 \times 15+1)$ pixels. Fitting is also local in time, typically limited to about a 100 frames.

\section{Experimental Results}

The preliminary results obtained by using both imaging techniques are now presented. DP600 steel produced by Arcelor (dual-phase carbon steel) was the material tested. Thin flat specimens were used with a gauge part of $10 \mathrm{~mm}$ (length), $10 \mathrm{~mm}$ (width) and $2.6 \mathrm{~mm}$ (thickness). This material is a hot-rolled steel grade containing ferrite and martensite. It is composed of $0.074 \mathrm{C}, 0.84 \mathrm{Mn}, 0.038 \mathrm{P}, 0.002 \mathrm{~S}$, $0.217 \mathrm{Si}, 0.04 \mathrm{Al}, 0.702 \mathrm{Cr}$, and $0.005 \mathrm{~N}$ (in wt.\%). Table 1 presents the thermomechanical properties of DP600: the mass density $\rho$, the heat capacity $C$, the isotropic heat conduction coefficient $k$, the linear thermal expansion coefficient $\alpha_{\mathrm{d}}$, the Young's modulus $E$, the yield strength $\sigma_{y 0.02}$, the ultimate strength $\sigma_{\mathrm{u}}$ and the fatigue limits $\Delta \sigma_{\infty}$ (obtained at $2 \times 10^{6}$ cycles) expressed in terms of stress range $\Delta \sigma$ for stress ratio $R_{\sigma}=\sigma_{\min } / \sigma_{\max }$ equal to -1 .

The tests involved loading blocks of $N_{c}=2400$ cycles performed at different $\Delta \sigma$ with $R_{\sigma}=-1$ and $f_{\mathrm{L}}=$ $30 \mathrm{~Hz}$. Between the fifth and the last five blocks, a block of 100000 cycles was performed in the maximal stress range (Figure 2). The short blocks were

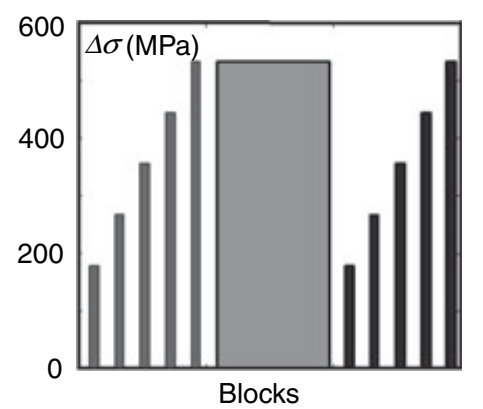

Figure 2: Series of blocks

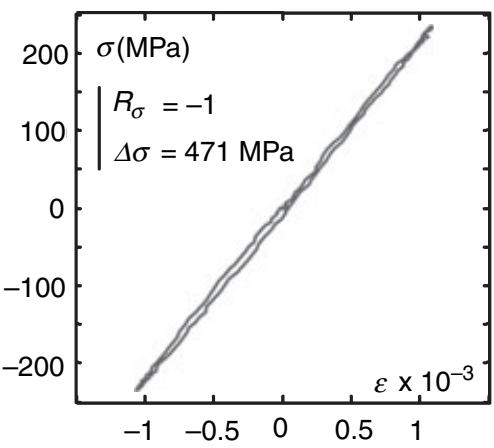

Figure 3: Example of stress-strain hysteresis loop

performed to estimate the dissipation levels at different stress amplitudes and at relatively constant damage state. Conversely, the long block at maximal constant stress range aimed at speeding up the fatigue development. The maximal stress range nearly corresponded to the fatigue limit. Note that the visible CCD camera data aquisitions were performed at $f_{\mathrm{L}}=5.55 \times 10^{-3} \mathrm{~Hz}$ to increase the $f_{\mathrm{S}} / f_{\mathrm{L}}$ ratio, with $f_{\mathrm{S}}$ denoting the sampling frequency, and to improve computation of the energy corresponding to the hysteresis area of the stress-strain curve (Figure 3). As calorimetric analysis showed that the energy dissipated in a cycle was independent of the loading frequency [17], we assumed that the deformation energy corresponding to the hysteresis loop of the stress-strain curve was also rate-independent. The loading block series of the fatigue test is schematically presented in Figure 2.

\section{Analysis of mechanical effects}

Figure 3 gives an example of a mean stress-strain hysteresis loop over the next to last block. Figure 4 shows time-course variations in the tensile strain

\begin{tabular}{|c|c|c|c|c|c|c|c|c|}
\hline$\rho\left(\mathrm{kg} \mathrm{m}^{-3}\right)$ & $C\left(\mathrm{~kg}^{-1}{ }^{\circ} \mathrm{C}^{-1}\right)$ & $K\left(\mathrm{~W} \mathrm{~m}{ }^{-1}{ }^{\circ} \mathrm{C}^{-1}\right)$ & $\alpha_{d}\left(10^{-6}{ }^{\circ} \mathrm{C}^{-1}\right)$ & $E(\mathrm{MPa})$ & $\sigma_{y 0.02}(\mathrm{MPa})$ & $\sigma_{u}(\mathrm{MPa})$ & $\Delta \sigma_{\infty}(\mathrm{MPa})$ & $R_{\sigma}$ \\
\hline 7800 & 460 & 64 & $|0-1|$ & 213000 & 360 & 611 & $526 \pm 5^{*}$ & -1 \\
\hline
\end{tabular}

Table I: Thermomechanical properties of DP600

*Standard deviation. 


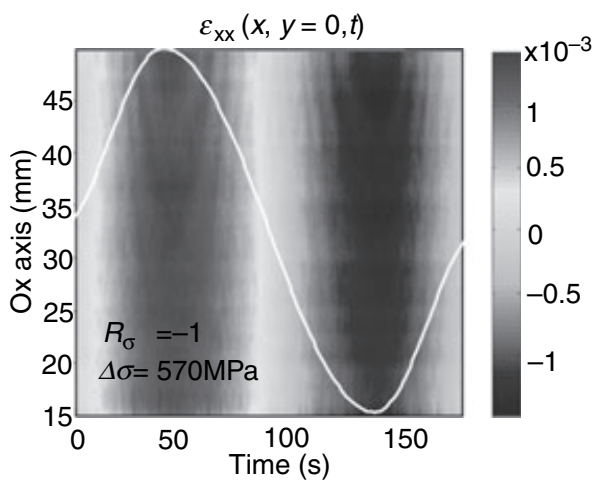

Figure 4: Time course of the tensile strain $\mathrm{O} x$ profile over a loading cycle

components $\varepsilon_{x x}$ captured along the longitudinal axis during a loading cycle performed at $\Delta \sigma=570 \mathrm{MPa}$. The white curve represents the sinusoidal load pattern. Analysis of the strain distributions enabled us to highlight small systematic strain gradients which were associated with the material heterogeneity.

Using the variational inverse method, the displacement fields obtained by DIC allowed us to estimate the elastic parameters of the steel [12]. Figures 5 and 6 show the different mean Young's modulus and Poisson's ratio values per block, for the 11 blocks, over the sample gauge part. These values can be compared with those obtained with the second method [13]. In this last case, the modulus was identified with the slope of the hysteresis loop. A linear regression was performed for each block and each couple $\left(\varepsilon_{x x}, \sigma_{x x}\right)$ of the strain and stress field measurements. The mean contraction coefficient was

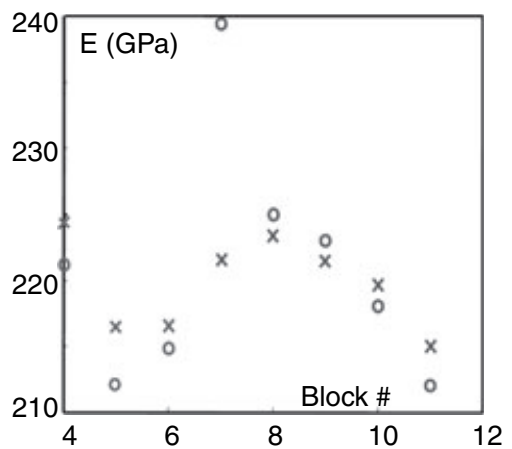

Figure 5: Mean Young's modulus values according to the block number

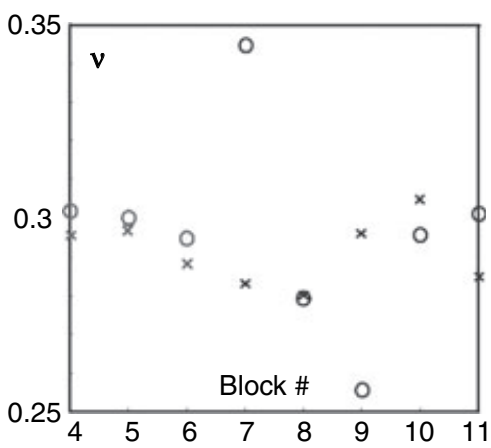

Figure 6: Mean Poisson's ratio values according to the block number

also directly derived from the strain field measurements by averaging the local and instantaneous $v(x, y, t)=-\varepsilon_{y y} / \varepsilon_{x x}$. The results in Figures 5 and 6 indicate that the elastic properties of the material remained almost constant throughout the 124000 cycles. The undetected influence of fatigue on the elastic parameters is quite surprising and interesting if fatigue mechanisms are considered in terms of damage combined with elasticity [18] and/or plasticity [19].

\section{Analysis of energy effects}

We used both stress and strain field measurements to compute the mean volume energy rate corresponding to the mean hysteresis area per block $A_{\mathrm{h}}$. Under the plane stress hypothesis, we computed:

$f_{\mathrm{L}} A_{\mathrm{h}}(x, y)=\frac{f_{\mathrm{L}}}{N_{\mathrm{c}}} \int_{0}^{N_{\mathrm{c}} f_{\mathrm{L}}^{-1}} \sigma_{i j}(x, y, t) \dot{\varepsilon}_{i j}(x, y, t) \mathrm{d} t$

Dividing $f_{\mathrm{L}} A_{\mathrm{h}}$ by the volume heat capacity $\rho C$ of the material, we obtained a mean deformation energy rate per cycle expressed in ${ }^{\circ} \mathrm{C} \mathrm{s}^{-1}$. This operation makes it possible to define an equivalent heating speed for each type of energy rate and facilitates comparison between the different terms of the energy balance.

The calorimetric terms were derived from the polynomial coefficients of $p_{i}(x, y)$. By denoting $P_{1}, \ldots$, $P_{24}$ as the fitting parameters, the corresponding expressions of $\tilde{\Delta} s_{\text {the }}$ and $\tilde{d}_{1}$ are:

$$
\frac{\tilde{\Delta} s_{\text {the }}}{\rho C}=2 \sqrt{\begin{array}{l}
\left(P_{1} \omega \frac{I^{2}}{N_{x}^{g}}+P_{5} \omega \frac{J^{2}}{N_{y}^{g}}+P_{21} \omega+\frac{1}{\tau_{\text {th }}^{2 D}}\left(P_{2} \frac{I^{2}}{N_{x}^{g}}+P_{6} \frac{J^{2}}{N_{y}^{g}}+P_{22}\right)-\frac{2 k}{\rho C}\left(\frac{P_{2}}{\Delta x^{2}}+\frac{P_{6}}{\Delta y^{2}}\right)\right)^{2} \\
+\left(-P_{2} \omega \frac{I^{2}}{N_{x}^{g}}-P_{6} \omega \frac{J^{2}}{N_{y}^{g}}-P_{22} \omega+\frac{1}{\tau_{\mathrm{th}}^{2 D}}\left(P_{1} \frac{I^{2}}{N_{x}^{g}}+P_{5} \frac{J^{2}}{N_{y}^{g}}+P_{21}\right)-\frac{2 k}{\rho C}\left(\frac{P_{1}}{\Delta x^{2}}+\frac{P_{5}}{\Delta y^{2}}\right)\right)^{2}
\end{array}}
$$




$$
\begin{aligned}
\frac{\tilde{d}_{1}}{\rho C} & =\left(\frac{P_{3}}{\Delta \tau}+\frac{P_{4}}{\tau_{\mathrm{th}}^{2 D}}\right) \frac{I^{2}}{N_{x}^{g}}+\left(\frac{P_{7}}{\Delta \tau}+\frac{P_{8}}{\tau_{\mathrm{th}}^{2 D}}\right) \frac{J^{2}}{N_{y}^{g}} \\
& +\left(\frac{P_{23}}{\Delta \tau}+\frac{P_{24}}{\tau_{\mathrm{th}}^{2 D}}\right)-\frac{2 k}{\rho C}\left(\frac{P_{4}}{\Delta x^{2}}+\frac{P_{8}}{\Delta y^{2}}\right)
\end{aligned}
$$

where $N_{x}^{g}=2 N_{x}+1$ and $N_{y}^{g}=2 N_{y}+1$ are the number of pixels of the fitting window,

$I^{2}=\sum_{i=-N_{x}}^{N_{x}} i^{2}$ and $J^{2}=\sum_{i=-N_{y}}^{N_{y}} j^{2}$,

$\Delta x$ and $\Delta y$ are the space resolutions, $\Delta \tau$ is the time step associated with the frame rate (i.e. the sampling frequency $f_{\mathrm{S}}$ ), and $f_{\mathrm{L}} / f_{\mathrm{S}}$. A detailed presentation of the image processing and its check should be available in the near future [17].

In Figure 7, we presented a 2-D distribution of the mean thermoelastic source amplitudes averaged over the last loading block. The two main features to be noted are the quite good homogeneity of the coupling source field and the order of magnitude of the thermoelastic effects. The mean $\tilde{\Delta} s_{\text {the }}$ is around $92.5^{\circ} \mathrm{C} \mathrm{s}^{-1}$ with a standard deviation of about $0.3{ }^{\circ} \mathrm{C} \mathrm{s}^{-1}$. Note that the amplitude $\tilde{\Delta} s_{\text {the }}$ is much higher than the dissipation intensity. The energy dissipated per cycle was also compared with the anelastic energy corresponding to the hysteresis loop.

In Figure 8, the average longitudinal dissipation profile corresponding to the last block can be compared with $A_{\mathrm{h}}^{(1)}$, which is the anelastic energy rate profile derived from the variational approach. We also plotted the $A_{\mathrm{h}}^{(2)}$ profile obtained using the second method. In both cases, we observed that the anelastic work was higher than the energy dissipated in a cycle. We finally plotted the 'initial' width variations $w_{0}(x)$ of the specimen to show the reader

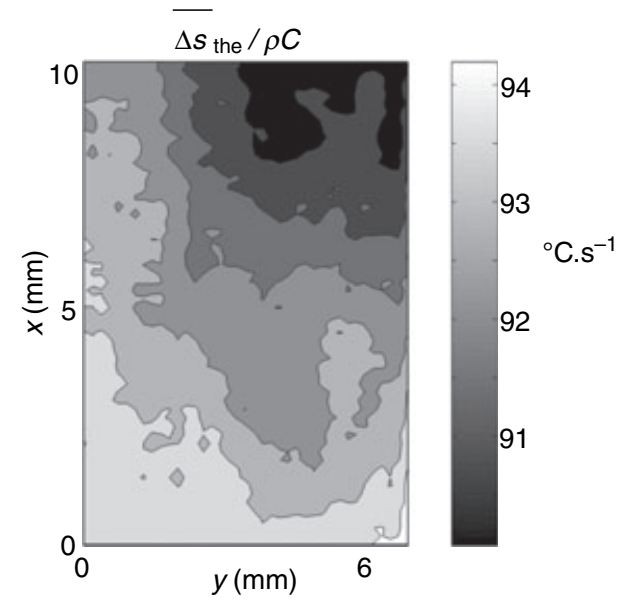

Figure 7: Mean 2-D distribution of thermoelastic sources $\tilde{\Delta} s_{\text {the }}(x, y)$ corresponding to the last loading block

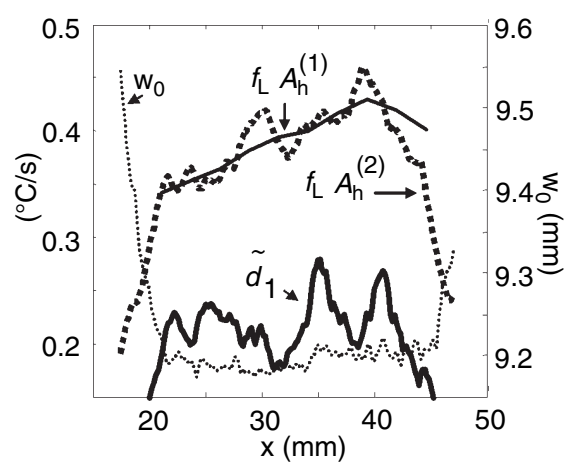

Figure 8: Mean profile over the sample width of $f_{\mathrm{L}} A_{\mathrm{h}}^{1}, f_{\mathrm{L}} A_{\mathrm{h}}^{2}$ and $\tilde{d}_{1}$

the limits of the gauge part and the sample geometry. Here, by 'initial' we mean prior to the observed block.

In Figure 9, the energy balance was performed for all blocks by considering the mean anelastic energy (using both methods) and dissipated energy values over the sample gauge part. This result deserves some comment because the hysteresis area is often identified with the dissipated energy. Let us consider the local expressions of both principles of thermodynamics:

$\rho \dot{e}=\sigma: \dot{\varepsilon}-\operatorname{div} q+r_{\text {ext }}$

$\rho T \dot{s}-r_{\mathrm{ext}}+\operatorname{div} q=d_{1}$

where $e$ is the specific internal energy, $q=-K \operatorname{grad} T$ the heat influx vector and $s$ the specific entropy. By combining both equations, we get the following expression of the volume deformation energy rate:

$\sigma: \dot{\varepsilon}=\rho \dot{e}+d_{1}-\rho T \dot{s}=\rho \dot{e}+d_{1}-\rho C \dot{T}+s_{\text {the }}+s_{\text {ic }}$

Integrating Equation (14) over a mechanical cycle leads to the hysteresis area $A_{\mathrm{h}}$. If the mechanical cycle

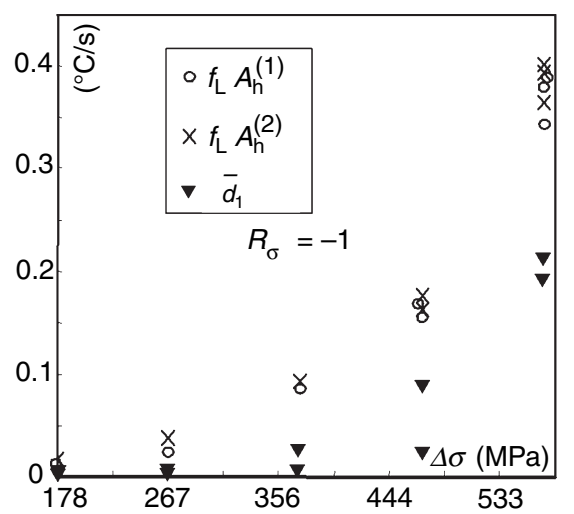

Figure 9: Mean $f_{\mathrm{L}} A_{\mathrm{h}}^{1}, f_{\mathrm{L}} A_{\mathrm{h}}^{2}$ and $\tilde{d}_{1}$ values over the gauge part and per block 
is also a thermodynamic cycle, the hysteresis area can come from intrinsic dissipation and/or thermomechanical coupling sources [20]. Concerning DP600 steel fatigue, we found that the variation in the thermoelastic energy $w_{\text {the }}$ vanished at the end of each loading cycle. Besides we neglected the internal coupling sources. The hysteresis area is thus caused by internal energy and dissipated energy variations as soon as a periodic oscillation of the temperature is reached (generally obtained after some hundreds of cycles). We then interpreted the difference between the energy of the hysteresis loop and the dissipated energy as an internal energy variation due to irreversible fatigue mechanisms. At this level we ought to mention the works of Kaleta [21], who showed similar results at the macroscopic scale (i.e. the scale of the specimen). Indeed, in the case of ferritic-perlitic steel, he observed stored energy ratios associated with the stress-strain hysteresis loop varying from $37.4 \%$ for low cycle fatigue tests (LCF), and up to $67.3 \%$ for high cycle fatigue tests (HCF). We also give references dealing with the fatigue of aluminium alloys for which stored energy ratios of about 0.8 to 0.9 were found [22, 23].

\section{Concluding Comments}

In this paper, we presented a combined application of DIC and IRT to the fatigue of DP600 steel. We used kinematic and thermal data to estimate the deformation energy rate, thermoelastic source amplitude and mean intrinsic dissipation per cycle. The deformation energy rate distribution was computed using strain field measurements, with the stress estimated using a variational inverse method. The heat source distributions were derived from thermal data using a local expression of the heat equation. The linearity of the diffusion equation and that of the boundary conditions allowed us to estimate the sources separately. Finally, the preliminary results obtained on DP600 steel were presented. These results did not reveal any noticeable influence of high cycle fatigue on the elastic properties (up to $10^{5}$ cycles). Moreover, they showed that the amounts of deformation energy corresponding to the stress-strain hysteresis loop were systematically greater than the dissipated energy per cycle. A thermodynamic analysis led us to interpret these differences as internal energy variations. This finding could obviously have a marked impact on the modeling of steel fatigue kinetics, so these preliminary results must, as usual, be considered with precaution. Complementary checks and tests should be performed in the near future. Never- theless, the promising results obtained with the local energy approach, combining DIC with IRT, is already very encouraging.

\section{ACKNOWLEDGEMENTS}

The authors would like to thank Arcelor Research SA and Nippon Steel Corporation for their technical and financial support during this study. The authors, members of LMGC, would also like to warmly thank Andre Galtier for his confidence over the last 10 years and for the fruitful and animated scientific discussions.

\section{REFERENCES}

1. Galtier, A. (1993) Contribution à l'étude de l'endommagement des aciers sous sollicitations uni ou multiaxiale. PhD Thesis, ENSAM, Bordeaux.

2. Luong, M. P. (1998) Fatigue limit evaluation of metals using an infrared thermographic technique. Mech. Mat. 28, 155-163.

3. LaRosa, G. and Risitano, A. (2002) Thermographic methodology for rapid determination of the fatigue limit of materials and mechanical components. Int. J. Fatigue 22, 65-73.

4. Liaw, Y. B., Wang, P. K., Jiang, L., Huang, J. Y., Kuo, R. C. and Huang, J. C. (2001) Thermographic investigation of the fatigue behaviour of reactor pressure vessel steels. Mat. Sci. Eng. A 314, 131-139.

5. Krapez, J. C., Pacou, D. and Gardette, G. (2000) Lock-in thermography and fatigue limit of metals. Proc. QIRT'2000, Reims, France, 277-282.

6. Krapez, J. C. and Pacou, D. (2002) Thermography detection of damage initiation during fatigue tests. Proc. SPIE Thermosense XXIV, Orlando, FL, 4710, 435-449.

7. Cugy, P. and Galtier, A. (2002) Microplasticity and temperature increase in low carbon steels. Proc. 8th Int. Fatigue Conf., Stockholm, Sweden, 549-556.

8. Mabru, C. and Chrysochoos, A. (2001) Dissipation et couplages accompagnant la fatigue des matériaux métalliques. Proc. Photomécanique' 01, Poitiers, France, 375-382.

9. Wattrisse, B., Chrysochoos, A., Muracciole, J.-M. and Nemoz-Gaillard, M. (2000) Analysis of strain localisation during tensile test by digital image correlation. J. Exp. Mech. 41, 29-38.

10. Germain, P., Nguyen, Q. S. and Suquet, P. (1983) Continuum thermodynamics. J. Appl. Mech. 50, 1010-1020.

11. Geymonat, G., Hild, F. and Pagano, S. (2002) Identification of elastics parameters by displacement field measurement. C.R. Mécanique 330, 403-408.

12. Latourte, F., Chrysochoos, A., Geymonat, G., Pagano, S. and Wattrise, B.2005) Full-field measurements applied to the identification of a non linear mechanical behaviour. Proc. Euromech Mecamat EMMC8, Cachan, France, 155-163.

13. Wattrisse, B., Chrysochoos, A., Muracciole, J.-M. and Nemoz-Gaillard, M. (2001) Kinematic manifestations of localisation phenomena by image intercorrelation. Eur. J. Mech. A/Solids, 20, 189-211. 
14. Chrysochoos, A., Muracciole, J.-M. and Wattrisse, B. (2000) Experimental analysis of strain and damage localization. Proc. Symp. Continuous Damage and Fracture, Cachan, France, 41-51.

15. Chrysochoos, A. and Louche, H. (2000) An infrared image processing to analyze the calorific effects accompanying strain localization. Int. J. Eng. Sci. 38, 1759-1788.

16. Boulanger, T., Chrysochoos, A., Mabru, C. and Galtier A. (2004) Calorimetric and thermoelastic effects associated with the fatigue behavior of steels. Int. J. Fatigue 26, 221-229.

17. Berthel, B., Galtier, A., Wattrisse, B. and Chrysochoos A. (2007) Thermographic analysis of fatigue dissipation properties of steel sheets. Strain 43, 273-279.

18. Lemaitre, J. (1992) Course on Damage Mechanics. Springer Verlag, Berlin.

19. Charkaluck, E. and Constantinescu, A. (2006) Estimation of thermoplastic dissipation in high cycle fatigue. C.R. Mécanique 334, 373-379.
20. Peyroux, R., Chrysochoos, A., Licht, C. and Löbel, M. (1998) Thermomechanical couplings and pseudoelasticity of shape memory alloys. Int. J. Eng. Sci. 36, 489-509.

21. Kaleta, J. (1998) Determination of cold work energy in LCF/HCF region. In: Proc. 4th Int. Conf. on Low Cycle Fatigue and Elastoplastic Behaviour, (K. T. Rie and P. D. Portella, Eds). Elsevier Science Ltd., Garmisch-Partenkirchen, 93-98.

22. Wong, A. K. and Kirby, G. C. III (1990) A hybrid numerical/experimental technique for determining the heat dissipated during low cycle fatigue. Eng. Fracture Mech. 37, 493-504.

23. Harvey, D. P. II and Bonenberger, R. J. Jr (2000) Detection of fatigue macrocracks in 1100 aluninium from thermomechanical data. Eng. Fracture Mech. 65, 609-620. 